\title{
CRMO: Case Series of an Orthopaedic Ambulatory
}

\author{
Magnani $\mathbf{M}^{*}$ \\ Orthopedics and Traumatology Pediatric Rizzoli \\ Orthopedic Institute, Italy \\ *Correspondling author: Marina Magnani, \\ Orthopedics and Traumatology Pediatric Rizzoli \\ Orthopedic Institute, Italy
}

Received: November 14, 2017; Accepted: March 23, 2018; Published: March 30, 2018

\begin{abstract}
Chronic Recurrent Multifocal Osteomyelitis (CRMO) is a disease rare autoimmune affecting mostly children between 10 and 12 years. Symptoms are often non-specific with laboratory tests initially blurred (VES PCR) or negative, children are often seen by different specialists before the actual diagnosis, thus delaying the therapy that if started promptly, it gives good results and reduces the risk of deformity for a long time term. The aim of the work is to describe a "case series" of 5 patients who have come to the attention in orthopedic surgery and the description of the symptoms e of the natural history of the disease.
\end{abstract}

Keywords: Chronic recurrent multifocal osteomyelitis; Orthopedic surgery; Auto inflammatory disease

\section{Introduction}

Chronic Recurrent Multifocal Osteomyelitis is an auto inflammatory rare disease that mostly affects children. We can find the causes of the disease into gene mutations in some cases (chromosome 18q) [1]. The disease affects more girls than boys and the peak for incidence is 10 years of age, even if symptoms can be present in each age.

\section{Symptoms}

CRMO is characterize by many symptoms such as deep aching pain, limping, tenderness and often fevers; they are in the same area or in different. The long bones of the body, the shoulder and collar bone, the spine, the ankles and the feet are common sites. Involvement of the sternum, clavicle, or mandible is particularly suggestive of CRMO. Patients can have also skin manifestations, such as psoriasis, acne, pustules on the hands and feet, or uveitis, inflammatory bowel disease. Majored syndrome, an auto inflammatory disease, seems CRMO, but it presents with symptoms of congenital dyserythropoietic anemia and Sweet Syndrome [2].

Unifocal CRMO can seem a tumor or an infection, for this reason a bone biopsy is mandatory to permorm diagnosis [3]. The lower limb did predominate as the initial site of bone lesions, but in general the join just near the bone is the first affected: ankle swelling is related to a neighboring tibial or fibular bone lesion, knee swelling is associated with a neighboring femoral lesion. The long bones are most frequently affected in lower-limb involvement Equating to $62 \%$ of lesions [4].

\section{Laboratory tests}

Two-thirds of patients have laboratory evidence of systemic inflammation with elevations in the erythrocyte sedimentation rate, C-Reactive Protein (CRP) level, leukocytes, and fibrinogen during the attacks of bone pain.

Thanks to the whole-body Magnetic Resonance Imaging (MRI) the diagnosis is easier. Radiography is the first-line imaging study in pediatric patients but they are often normal initially. However, in some times we can find some radiological findings such as breached cortices and/or periosteal appositions; these signs can suggest a tumor, particularly if the lesion is unifocal.
The main differential diagnoses are osteomyelitis, primary bone tumors, lymphomas, and Langerhans cell histiocytosis. The prevalence of spinal involvement varies considerably, from 3 to $25 \%$, depending on the study. Whole-body MRI is more sensitive than radionuclide bone scanning6. If clinically there is a real suspect of CRMO, Whole-body MRI with STIR sequences can substantially contribute to an early diagnosis: The typical pattern is a hyperintense multifocal and bilateral lesions bone involvement above all in lower extremities, pelvis, shoulder girdle

and spine, and shows their characteristic proximity to growth plates, sacroiliac joints and triradiate cartilage. Additionally, WBMRI may help to uncover asymptomatic vertebral compressions that are the best site for a biopsy [5]. The criteria to performed diagnosis are summarizing in Table 1.

\section{Treatment}

The treatment of CRMO is not standardized. Generally CRMO resolves without consequences, but recently authors suggest that physical impairments may persist in up to $50 \%$ of patients, such as chronic pain and bone deformities, sometimes with limb length abnormalities. 3 If vertebral bodies are involved permanent deformity of the spine are more often and more aggressive treatment is needed [6]. There no standardized treatment, however for about all authors NSAIDs are the accepted first-line medications. The second steps when NSAIDs are ineffective are bisphosphonates and TNF-antagonists. Other therapy with azithromycin, interferon, sulfasalazine, methotrexate, intravenous immunoglobulin's, and colchicine are described. 3 In Table 2 we summarize the most used medical therapies. The probably most effective therapy are oralglucocorticoids, but due to the adverse effects of long term therapy on growth they should be used only in limited dosages and durations. For other therapy in 2004 bisphosphonates are demonstrated effective, and for patients with a refractory form are recommended TNF-antagonists [3]. In some cases patients are treated with systemic antibiotics at the begin of the manifestation without any effect and, of course, cultures of bone are typically negative. Talking about the duration of the therapy, in a retrospective review 13 years after initial diagnosis, Huber, et al. described 23 patients; the results suggest that intermittent treatment of acute relapses is as effective as long-term 
Table 1: Diagnostic Criteria for Nonbacterial Osteitis/ Chronic Recurrent Multifocal Osteomyelitis.

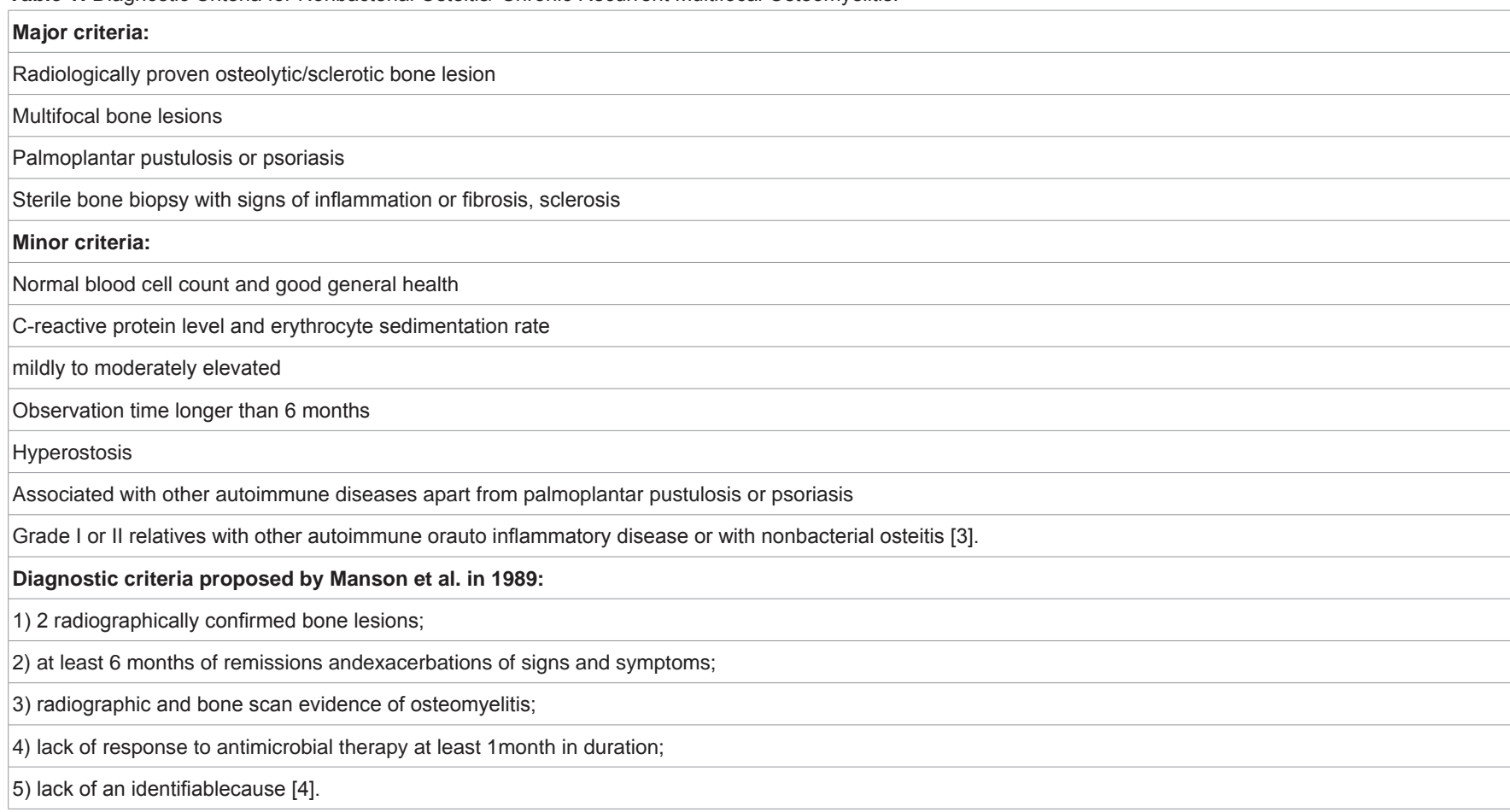

therapy [4-8].

\section{Materials and Methods}

\section{Case 1 (Villani)}

Female 10 years, sudden pain with stiff neck and Cock Robin Sign, after a slight trauma reported during normal activities, she was initially treated with manipulations. Then she was hospitalized after 2 months, due to the persistence of the rotary luxation of C1-C2. In the anamnesis the girl has reported also an initial difficulty walking with lameness already a few weeks before the trauma. She has been studied with RX, NMR and CT scan that showed a cervical rotatory luxation type 2 [1], there were very mild aspects of inflammation, type chronic process $\mathrm{C} 1-\mathrm{C} 2$ in NMR, but the PET showed hyperfixation C1-C2 and acetabulum, the sacroiliac joint and the sternoclavicular. Given the nature of the clinical-radiographic definitely not posttraumatic, we decided for conservative treatment. After the evaluation at the Pediatric Rheumatic center where Chronic Recurrent Multifocal Osteomyelitis (CRMO) was diagnosed, we changed the initial indication with halo-jacket and progressive traction with chin traction and Philadelphia Collar. After the correct diagnosis, we have undertaken progressive traction with chin and physiotherapy along with specific medical therapy for the rheumatologic disease with bisphosphonates and immunosuppressant's. The clinical response has been good. At last 2-year follow-up MRI, inflammation appears off in all districts also shows a partial correction of luxation C1-C2.

\section{Case 2 (Cizmi)}

A ten years old female comes to our attention with swelling to her right collarbone. In clinical history we found a only a dental abscess episode a few months before. At clinical examination we found only local fixed tough swelling, no pain or local sign of flogosis; no similar cases in her family. After the clinical evaluation we perform an X-ray
Table 2: Reported Medical Therapies for CRMO.

\begin{tabular}{|l|}
\hline Antiinflammatory \\
\hline Nonsteroidal antiinflammatory drugs \\
\hline Immune modulator \\
\hline Corticosteroids \\
\hline Interferon gamma \\
\hline Interferon alpha \\
\hline Intravenous immunoglobulin \\
\hline Sulfasalazine \\
\hline Dapsone \\
\hline Antimetabolite \\
\hline Methotrexate \\
\hline Calcium modulator \\
\hline Bisphosphonate \\
\hline Other \\
\hline Colchicine \\
\hline Hyperbaric oxygen \\
\hline Combinations \\
\hline Azithromycin - calcitonin - bisphosphonate \\
\hline Diclofenac - prednisolone [5]. \\
\hline
\end{tabular}

that showed a cortical irregularity with bone remodeling. CT scan confirmed bone alteration without a real local lesion. During the hospitalization the girl complained back pain: scintigraphy showed multiple capitations in the collarbone and thoracic vertebrae and the spine MRI showed rehash of bone structure of multiple vertebrae and wedging of thoracic ones (T3,T7,T8,T9,S1,S4). The wedge deformity of T3 need for treatment with high collar with sternal support. 
PCR and VES were very high (1.86 n.v 0.5 and 71 n.v <50), WBC were slightly increased. We performed an incisional biopsy with clinical suspected diagnosis of leukemia or X-histiocytosis. The biopsy revealed: chronic osteomyelitis with negative bacterial culture. We contacted the colleague of Infective diseases that ordered thighs tang legs RMI with the suspect of CRMO. After the findings of bone remodeling with a flogosis signal in the two ankles we transferred the young patient in Pediatric Rheumatology Department where started the therapy (bisphosphonates and corticosteroids). After that the disease went into complete remission.

\section{Case 3 (Lo Forte Benedetta)}

First orthopaedic visit performed at 10 years old for right hip pain. Clinical examination showed only a limitation of the Range of motion for pain in the right hip at the maximum degrees. The RMI performed during the hospitalization showed only an enlargement of the inguinal lymph nodes. WBC was 10.000. After few months a tibial fracture occurred, treated by a long cast. After the fracture resolution the hip pain returned more and more painful with limping and reduction of the calcic density at the $\mathrm{x}$-rays. Then the girl complained neck and knee pain. The total-body MRI showed an iper-intensive signal of the femur and the tibia. We performed the bone biopsy in the femur that confirms the hypothesis of CRMO.

\section{Conclusion}

CRMO is a childhood-onset disease that can substantially impair quality of life by inducing persistent symptoms or sequelae. Although this chronic disease is not exceedingly rare, it remains poorly recognized by physicians and its prevalence is probably under estimated. Chronic recurrent multifocal Osteomyelitis is a not frequently observed pathology and is not strictly relevant orthopedic. Is a skeletal disorder of unknown etiology mainly occurring in children and adolescents? From a clinical point of view at the beginning th CRMO can mime a septic state, with high fever, general impairment and alteration of the flogosi parameters ( VES PCR fibrinogen) or most frequently, the main symptom is a persistent and recurrent pain, due to a trauma or a fracture, with non-specific laboratory findings, however, raises the suspicion of evaluating a different diagnosis, because there are non classic signs of acute infections like swelling, heat, functional limitation of neighboring joints, but there are an unusual location of the lesions, versus Osteomyelitis, with a frequent involvement of clavicle, tubular bones of the lower limbs and spine.

The different diagnosis mainly includes Osteomyelitis and tumor or a tumor like lesions, jovenile idiopathic arthritis. Biopsy may be needed to exclude by histological examination the rare case of Ewing sarcoma or leukemia or histiocytosis X/ eosinofil granuloma, and provide the material for microbiological studies. But often histological finding is non specific, compatible with subacute or chronic Osteomyelitis.

From the instrument poin of view rx is initially negative them comes a deteriorations of the bone marrow signal only detectable by MRI, wich shows areas of transparency like "map" interesting the medullary space symmetrically. In acute osteomielities there is no symmetrical distribuction, the location of the lesion tends to be at metafisys and is rounded with margins increasing in evolution of disease. The Lesion in CRMO is predominantly located on tubular bones followed by clavicle, the spine and pelvic bones. The lesions are often multiple and sometimes symmetrical. The diagnosis is usually based on the characteristic course and conventional radiographic findings supported by MRI and histopathological examination. The CRMO not responding to antibiotics therapy and cortisone. In conclusion CRMO has been conjecture to be an autoimmune disorder, (it has been reported to be associated with several autoimmune disease like a syeronegativ jovanile form of spondiloartritis and a psoriasis) it seems to be an autoimmune inflammatory reaction as a response to an infection comparable to the reaction occurring in arthritis; the occasional occurrence of CRMO exacerbations in connection with common infections disease supports this. This condition first describe in Europe by Giedion, affects females more often than males and occurs predominantly in children; CRMU is still diagnosed primarily by exclusion of other conditions and often only on long term followup, and it is based on clinical and radiological data, in most cases biopsy and bone histology can confirm the diagnosis.

In case of cervical spine injuries diagnosis and functional outcome especially for very young children are difficult to manage. The presence of a modest trauma with rotary dislocation makes necessary a deep clinical examination and evaluation of co morbidities (Case 1). The cervical trauma in childhood is often under or overestimated and in case of minor trauma some other causes (rheumatology, inflammatory) should always be investigated starting from the history, considering the existence of other signs and symptoms of disease [9-11]

\section{References}

1. Golla A1, Jansson A, Ramser J, Hellebrand H, Zahn R, Meitinger $\mathrm{T}$, Belohradsky BH, Meindl A, et al. Chronic recurrent multifocal osteomyelitis (CRMO): evidence for a susceptibility gene located on chromosome 18q21.318q22. Eur J Hum Genet. 2002; 10: 217-221

2. Roderick MR, Shah R, Rogers V, Finn A, Ramanan AV. Chronic recurrent multifocal osteomyelitis (CRMO) - advancing the diagnosis. Pediatr Rheumatol Online J. 2016; 14: 47.

3. Wipff J1, Adamsbaum C, Kahan A, Job-Deslandre C. Chronic recurrent multifocal osteomyelitis. Joint Bone Spine. 2011; 78: 555-560.

4. Ferrari J, Pilkington C. Chronic recurrent multifocal osteomyelitis: the prevalence of lower-limb and foot involvement. J Am Podiatr Med Assoc. 2014; 104: 583-587.

5. Chun CS. Chronic recurrent multifocal osteomyelitis of the spine and mandible: case report and review of the literature. Pediatrics. 2004; 113: e380-384.

6. Ferguson PJ, Sandu M. Current understanding of the pathogenesis and management of chronic recurrent multifocal osteomyelitis. Curr Rheumatol Rep. 2012; 14: 130-141.

7. Barrani M, Massei F, Scaglione M, Paolicchi A, Vitali S, Ciancia EM, et al. Unusual onset of a case of chronic recurrent multifocal osteomyelitis. Pediatr Rheumatol Online J. 2015; 13: 60

8. Von Kalle T, Heim N, Hospach T, Langendörfer M, Winkler P, Stuber T. Typical patterns of bone involvement in whole-body MRI of patients with chronic recurrent multifocal osteomyelitis (CRMO). Rofo. 2013; 185 : 655-661.

9. Greggi T, Scarale A, Maredi E, Lolli F, Martikos K, et al. The Cervical Trauma in Children: Difficulties in Diagnosis and Treatment Choice. J Spine 4: 214.

10. Wipff J, Adamsbaum C, Kahan A, Job-Deslandre C. Chronic recurrent multifocal osteomyelitis. Joint Bone Spine. 2011; 78: 555-560.

11. Hedrich CM, Hofmann SR, Pablik J, Morbach H, Girschick HJ. Autoinflammatory bone disorders with special focus on chronic recurrent multifocal osteomyelitis (CRMO). Pediatr Rheumatol Online J. 2013; 11: 47. 